\title{
Tanshinone I, an Active Ingredient of Salvia miltiorrhiza, Inhibits Differentiation of 3T3-L1 Preadipocytes and Lipid Accumulation in Zebrafish
}

\author{
Hyo-Shin Kwon, Byeong-Churl Jang \\ Department of Molecular Medicine, College of Medicine, Keimyung University
}

Received: December 8, 2020

Revised: December 13, 2020

Accepted: December 14, 2020
Correspondence to: Byeong-Churl Jang Department of Molecular Medicine, College of Medicine, Keimyung University, 1095 Dalgubeoldaero, Dalseo-gu, Daegu 42601, Korea.

Tel: +82-53-258-7404

Fax: +82-53-258-7403

E-mail: jangbc123@gw.kmu.ac.kr

Copyright $\odot 2020$ by The Society of Korean Medicine for Obesity Research
Objectives: Tanshinone I is a bioactive constituent in Salvia miltiorrhiza. At present, the anti-obesity effect and mechanism of tanshinone I are not fully understood. Here we investigated the effect of tanshinone I on lipid accumulation in 3T3-L1 preadipocytes and zebrafish.

Methods: Lipid accumulation and triglyceride (TG) content in 3T3-L1 cells were determined by Oil Red $\mathrm{O}$ staining and AdipoRed assay, respectively. The expression and phosphorylation levels of adipogenic/lipogenic proteins in 3T3-L1 cells were evaluated by Western blotting. The messenger RNA (mRNA) expression levels of adipogenic/lipogenic markers and leptin in 3T3-L1 cells were measured by reverse transcription polymerase chain reaction (RT-PCR). Lipid accumulation in zebrafish was assessed by LipidGreen2 staining.

Results: Tanshinone I at $5 \mu \mathrm{M}$ largely blocked lipid accumulation and reduced TG content in differentiating 3T3-L1 cells. Furthermore, tanshinone I decreased the expression of CCAAT/enhancer-binding protein- $\alpha(\mathrm{C} / \mathrm{EBP}-\alpha)$, peroxisome proliferator-activated receptor- $\gamma$ (PPAR $-\gamma)$, fatty acid synthase (FAS), acetyl CoA carboxylase (ACC), and perilipin $A$ but also the phosphorylation of signal transducer and activator of transcription-3 (STAT-3) in differentiating 3T3-L1 cells. In addition, tanshinone I increased the phosphorylation of adenosine 3',5'-cyclic monophosphate (cAMP)-activated protein kinase (AMPK) while decreased the intracellular adenosine triphosphate (ATP) content with no change in the phosphorylation and expression of liver kinase-B1 in differentiating 3T3-L1 cells. Importantly, tanshinone I also reduced the extent of lipid deposit formation in developing zebrafish.

Conclusions: These findings demonstrate that tanshinone I has strong anti-adipogenic effects on 3T3-L1 cells and reduces adiposity in zebrafish, and these anti-adipogenic effect in 3T3-L1 cells are mediated through control of C/EBP- $\alpha$, PPAR- $\gamma$, STAT-3, FAS, ACC, perilipin A, and AMPK.

Key Words: Tanshinone, Adipogenesis, AMP-activated protein kinases, 3T3-L1 cells, Zebrafish

\section{Introduction}

Obesity is a serious public health epidemic with about 2 billion adults overweight or obese. The association between obesity and adverse health consequences, including hyperlipidemia, cardiovascular disease, type 2 diabetes, and cancer, is well established ${ }^{1)}$. As a result, obesity is now among the leading factors for global morbidity and mortality ${ }^{2)}$. Increasing evidence also indicates that obesity is induced as a result of excessive adipocyte differentiation, also known as adipogenesis, in the adipose tissues and adipocytes ${ }^{3,4)}$. Any compound that inhibits excessive adipogenesis could therefore be a potential therapeutic option for obesity.

Adipogenesis is a biological process characterized by 
morphological, cellular, and biochemical changes ${ }^{4)}$. The differentiation process requires fibroblast-like preadipocytes to develop into mature adipocytes ${ }^{3)}$ under the control of adipogenesis-related transcription factors, including the family of CCAAT/enhancer-binding proteins (C/EBPs), peroxisome proliferator-activated receptors (PPARs), and signal transducer and activator of transcription (STAT) proteins ${ }^{5-8)}$, protein kinase A, extracellular signal-regulated protein kinase-1/2 (ERK-1/2), adenosine 3',5'-cyclic monophosphate $(\mathrm{cAMP})^{9-11)}$, lipogenic enzymes, such as fatty acid synthase (FAS) and lipid droplet (LD) associated proteins like perilipin $\mathrm{A}^{12,13)}$, and cAMP-activated protein kinase (AMPK) and its downstream effector acetyl CoA carboxylase (ACC) $)^{14-17)}$. Central to the early stage of 3T3-L1 adipocyte differentiation is the expression and phosphorylation of STAT-3, which then induces C/EBP- $\beta$ transcription ${ }^{6,18)}$.

The potential application of natural products as traditional herbal medicines for the treatment of obesity by reducing lipid accumulation, adipocyte cell size and/or stimulating lipid metabolism are well documented ${ }^{19)}$. Tanshinone I is a bioactive constituent among the tanshinones found in a traditional herbal medicine Red sage, also called Salvia miltiorrhiza. Recently, Red sage and its bioactive phytochemicals have attracted much attention for their pharmacological effects, including anti-inflammatory, anti-diabetic, anti-cancer, and cardio-cerebrovascular protective activities $^{20,21)}$. We have recently demonstrated that tanshinone IIA, one of the tanshinones in Red sage, greatly inhibits adipogenesis in 3T3-L1 cells as well as in zebrafish ${ }^{22)}$. This research led us to hypothesize that tanshinone I might have similar or potentially superior anti-adipogenic effects.

The present study examined the ability of tanshinone I to regulate lipid accumulation in 3T3-L1 adipocytes and zebrafish. We report that tanshinone I strongly inhibits lipid accumulation in 3T3-L1 cells and zebrafish, and its anti-adipogenic/lipogenic effect on 3T3-L1 cells is associated with dramatic changes in the expression or activation status of central adipogenic program mediators C/EBP- $\alpha$, PPAR- $\gamma$, FAS, ACC, perilipin A, STAT-3, and AMPK.

\section{Materials and Methods}

\section{Materials}

Polyclonal C/EBP- $\alpha$ antibody, monoclonal PPAR $-\gamma$ antibody, monoclonal STAT-3 antibody, monoclonal phospho-STAT-3 (p-STAT-3) antibody, polyclonal STAT-5 antibody and polyclonal phospho-STAT-5 (p-STAT-5) antibody were from Santa Cruz Biotechnology (Delaware, CA, USA). Polyclonal p-AMPK antibody, monoclonal AMPK antibody, polyclonal p-ACC antibody, polyclonal ACC antibody, polyclonal phospho-liver kinase-B1 (p-LKB-1) antibody, and polyclonal LKB-1 antibody were from Cell Signaling Technology (Danvers, MA, USA). Monoclonal FAS antibody was from BD Bioscience (San Jose, CA, USA). Tanshinone I was purchased from Selleckchem (Houston, TX, USA). Tanshinone I was prepared as a $10 \mathrm{mM}$ stock solution in dimethyl sulfoxide (DMSO). Monoclonal $\beta$-actin antibody was from Sigma (St. Louis, MO, USA). Polyclonal perilipin A antibody was from Bio Vision (Milpitas, CA, USA). Unless stated all other reagents were from Sigma.

\section{Differentiation of 3T3-L1 preadipocytes}

3T3-L1 preadipocytes (ATCC, Manassas, VA, USA) were grown in Dulbecco's modified Eagle's medium (DMEM) supplemented with $10 \%$ fetal bovine serum (FBS; Gibco, Rockville, MD, USA) and penicillin-streptomycin (Welgene, Daegu, Korea). Cells were grown to confluence and then incubated for a further 2 days before differentiation was induced. Differentiation was induced by incubating 3T3-L1 preadipcoytes with DMEM supplemented with 10\% FBS (Welgene) plus a cocktail of hormones (MDI): $0.5 \mathrm{mM}$ IBMX (M), $0.5 \mu \mathrm{M}$ dexamethasone (D), and $5 \mu \mathrm{g} / \mathrm{mL}$ insulin (I) in the absence (vehicle control; DMSO, 0.1\%) or presence of tanshinone $\mathrm{I}$ at the indicated concentrations. After $48 \mathrm{~h}$ MDI-induction, the differentiation medium was replaced with DMEM supplemented with $10 \%$ FBS and 5 $\mu \mathrm{g} / \mathrm{mL}$ insulin in the presence or absence of tanshinone I at the indicated concentrations. The cells were then fed every other day with DMEM containing 10\% FBS in the presence or absence of tanshinone I at the indicated concentrations 
until day 8. On day 8 , the preadipocytes became mature adipocytes that rounded-up and filled with many oil droplets.

\section{Oil Red 0 staining}

On day 8 of differentiation, vehicle control (DMSO) or tanshinone I-treated 3T3-L1 cells were washed twice with phosphate buffer saline (PBS), fixed with $10 \%$ formaldehyde for $2 \mathrm{~h}$ at room temperature (RT), washed with $60 \%$ isopropanol and dried completely. The fixed cells were then stained with Oil red $\mathrm{O}$ working solution for $1 \mathrm{~h}$ at RT and then washed twice with distilled water. In addition, quantitation of Oil red $\mathrm{O}$ staining was determined by Image J (U.S. National Institutes of Health, Bethesda, MD, USA). Lipid droplets were also observed by light microscopy (Nikon, Tokyo, Japan).

\section{Cell count analysis}

Vehicle or tanshinone I-treated 3T3-L1 cell viability was assessed by trypan blue exclusion assay. Viable cells were counted using phase contrast microscopy in triplicate. Data are mean \pm standard error (SE) of three independent experiments. Survival was expressed as a percentage of vehicle control.

\section{Quantification of cellular triglyceride (TG) content by AdipoRed assay}

Intracellular lipid content of vehicle or tanshinone I-treated 3T3-L1 cells were measured using a commercially available AdipoRed Assay Reagent kit according to the manufacturer's instructions (Lonza, Walkersville, MD, USA). After a $10 \mathrm{~min}$ incubation, fluorescence was measured on a Victor3 (Perkin Elmer Inc., Waltham, MA, USA) with excitation at $485 \mathrm{~nm}$ and emission at $572 \mathrm{~nm}$. TG content was expressed as a percentage of vehicle control.

\section{Preparation of whole cell lysates}

At the designated time points, vehicle or tanshinone I-treated 3T3-L1 cells were washed twice with PBS and proteins extracted using a modified RIPA buffer $(50 \mathrm{mM}$ Tris-Cl [pH 7.4], $150 \mathrm{mM} \mathrm{NaCl}, 0.1 \%$ sodium dodecyl sulfate, $0.25 \%$ sodium deoxycholate, $1 \%$ Triton X-100, $1 \%$
Nonidet P-40, $1 \mathrm{mM}$ ethylenediaminetetraacetic acid (EDTA), $1 \mathrm{mM}$ ethylene glycol tetraacetic acid (EGTA), proteinase inhibitor cocktail $[1 \mathrm{x}])$. The cell lysates were collected and centrifuged at $12,000 \mathrm{rpm}$ for $20 \mathrm{~min}$ at $4^{\circ} \mathrm{C}$. The supernatants were saved and protein concentrations determined by Bradford reagent (Bio-Rad, Hercules, CA, USA).

\section{Western blot analysis}

Proteins $(50 \mu \mathrm{g})$ were separated by sodium dodecyl sulfate-poiyacrylmide gel eletrophoresis (SDS-PAGE) (10\%) and transferred onto nitrocellulose membranes (Millipore, Burlington, MA, USA). The membranes were washed with TBS (10 mM Tris, $150 \mathrm{mM} \mathrm{NaCl}$ ) with $0.05 \%$ (vol/vol) tween 20 (TBST) followed by blocking with TBST containing $5 \%(\mathrm{w} / \mathrm{v})$ non-fat dried milk. The membranes were incubated overnight with antibodies specific for C/EBP- $\alpha$ $(1: 1,000)$, PPAR- $\gamma(1: 1,000)$, STAT-3 $(1: 2,000)$, p-STAT-3 (1:2,000), STAT-5 (1:2,000), p-STAT-5 (1:2,000), p-LKB-1 (1:1,000), LKB-1 (1:1,000), p-ACC $(1: 2,000), \quad$ ACC $(1: 2,000)$, FAS $(1: 1,000)$, perilipin $\mathrm{A}(1: 2,000)$ or $\beta$-actin $(1: 10,000)$ at $4^{\circ} \mathrm{C}$. The membranes were then exposed to secondary antibodies coupled to horseradish peroxidase for $2 \mathrm{~h}$ at RT. The membranes were washed three times with TBST at RT. Immunoreactivities were detected by enhanced chemiluminescence (ECL) reagents. Equal protein loading was assessed by the expression levels of $\beta$-actin protein.

\section{Reverse transcription polymerase chain reaction (RT-PCR) analysis}

At the designated time point, total cellular RNA in vehicle or tanshinone I-treated 3T3-L1 cells was isolated with the RNA-zol-B (Tel-Test, Friendswood, TX, USA). Three micrograms of total RNA were reverse transcribed using a random hexadeoxynucleotide primer and reverse transcriptase. Single stranded cDNA was amplified by PCR with the following primers. Primer sequences used are listed in Table 1. The PCR conditions applied were: C/EBP- $\alpha, 30$ cycles of denaturation at $95^{\circ} \mathrm{C}$ for $30 \mathrm{~s}$, annealing at $62^{\circ} \mathrm{C}$ for $30 \mathrm{~s}$, and extension at $72^{\circ} \mathrm{C}$ for $30 \mathrm{~s}$; PPAR- $\gamma, 30$ cycles of denaturation at $95^{\circ} \mathrm{C}$ for $30 \mathrm{~s}$, annealing at $53^{\circ} \mathrm{C}$ for $30 \mathrm{~s}$, and 
Table 1. Primer Sequences Used for RT-PCR

\begin{tabular}{lll}
\hline Gene & & \multicolumn{1}{c}{ Sequence } \\
\hline C/EBP- $\alpha$ & Sense & $5^{\prime}$-TTACAACAGGCCAGGTTTCC-3' \\
PPAR- $\gamma$ & Antisense & $5^{\prime}$-CTCTGGGATGGATCGATTGT-3' \\
& Sense & $5^{\prime}$-GGTGAAACTCTGGGAGATTC-3' \\
FAS & Antisense & $5^{\prime}$-CAACCATTGGGTCAGCTCTC-3' \\
& Sense & $5^{\prime}-$ TTGCTGGCACTACAGAATGC-3' \\
Perilipin A & Antisense & $5^{\prime}$-AACAGCCTCAGAGCGACAAT-3' \\
& Sense & $5^{\prime}$-CTTTCTCGACACACCATGGAAACC-3' \\
Leptin & Antisense & $5^{\prime}$-CCACGTTATCCGTAACACCCTTCA-3' \\
& Sense & $5^{\prime}$-CCAAAACCCTCATCAAGACC-3' \\
ACC & Antisense & $5^{\prime}$-CTCAAAGCCACCACCTCTGT-3' \\
& Sense & $5^{\prime}$-GTCAGCGGATGGGCGGAATG-3' \\
Actin & Antisense & $5^{\prime}$-CGCCGGATGCCATGCTCAAC-3' \\
& Sense & $5^{\prime}$-TCATGAAGTGTGCGTTGACATCCGT-3' \\
\hline
\end{tabular}

RT-PCR: reverse transcription polymerase chain reaction, C/EBP- $\alpha$ : CCAAT/enhancer-binding protein- $\alpha$, PPAR- $\gamma$ : peroxisome proliferator-activated receptor- $\gamma$, FAS: fatty acid synthase, ACC: acetyl CoA carboxylase.

extension at $72^{\circ} \mathrm{C}$ for $30 \mathrm{~s}$; FAS, 30 cycles of denaturation at $95^{\circ} \mathrm{C}$ for $15 \mathrm{~s}$, annealing at $55^{\circ} \mathrm{C}$ for $40 \mathrm{~s}$, and extension at $68^{\circ} \mathrm{C}$ for $45 \mathrm{~s}$; perilipin $\mathrm{A}, 30$ cycles of denaturation at $95^{\circ} \mathrm{C}$ for $1 \mathrm{~min}$, annealing at $55^{\circ} \mathrm{C}$ for $1 \mathrm{~min}$, and extension at $72^{\circ} \mathrm{C}$ for $1 \mathrm{~min}$; leptin, 30 cycles of denaturation at $95^{\circ} \mathrm{C}$ for $1 \mathrm{~min}$, annealing at $57^{\circ} \mathrm{C}$ for $1 \mathrm{~min}$, and extension at $72^{\circ} \mathrm{C}$ for $1 \mathrm{~min}$; ACC, 30 cycles of denaturation at $95^{\circ} \mathrm{C}$ for $1 \mathrm{~min}$, annealing at $62^{\circ} \mathrm{C}$ for $1 \mathrm{~min}$, and extension at $72^{\circ} \mathrm{C}$ for $1 \mathrm{~min} ; \beta$-actin, 25 cycles of denaturation at $95^{\circ} \mathrm{C}$ for 30 $\mathrm{s}$, annealing at $57^{\circ} \mathrm{C}$ for $30 \mathrm{~s}$, and extension at $72^{\circ} \mathrm{C}$ for 1 min. Expression levels of $\beta$-actin messenger RNA (mRNA) were used as an internal control to evaluate the relative mRNA expression of adipocyte-specific genes and leptin.

\section{Measurement of intracellular adenosine triphos- phate (ATP) levels}

3T3-L1 preadipocytes were seeded in 96-well plates and grown in the presence of differentiation media in the absence or presence of tanshinone I or 2-deoxyglucose (2-DG). On day 2, 5, and 8 of differentiation, intracellular ATP levels were measured by luciferase activity using a luminescence assay kit according to the manufacturer's protocol (ATPLite-1step; Perkin Elmer Inc.). After 2 min incubation, luminescence was measured on a Victor3 (Perkin Elmer Inc.).

\section{Zebrafish experiment}

A number of studies have demonstrated that zebrafish can be used to study lipid biology including lipid accumulation and metabolism ${ }^{22,23)}$. We and others also have recently shown that LipidGreen2 selectively stains lipid deposits in live zebrafish ${ }^{22,24,25)}$. Zebrafish was maintained under standard conditions as previously described ${ }^{26)}$. All experimental protocols involving zebrafish were approved by the Animal Care and Committee of the Korea Research Institute of Chemical Technology. Two dpf (day post fertilization) larvae were arrayed in 24-well plates in which each well contains 10 larvae $(\mathrm{n}=10)$ and $1 \mathrm{~mL}$ egg water $(0.6 \mathrm{~g} / \mathrm{L}$ sea salt). The indicated concentrations of tanshinone I dissolved in DMSO were added to each well and incubated in $37^{\circ} \mathrm{C}$ incubator. After $24 \mathrm{~h}$, larvae were washed with egg water and stained with $5 \mu \mathrm{M}$ LipidGreen2 solution ${ }^{24)}$. After 10 min, larvae were washed 3 times with egg water. To acquire images, larvae were anesthetized in tricaine (Sigma) and mounted in 3\% methylcellulose (Sigma). Images were acquired with a Leica MZ10 F stereomicroscope, Leica DFC425 camera, and Leica Application Suite software v4.5 (Leica 
Microsystems, Wetzlar, Germany). Yolk size and fluorescence intensity were measured by ImageJ software ${ }^{27)}$. Data are mean \pm standard error of the mean.

\section{Statistical analysis}

Cell count analysis was done in triplicates and repeated three times. Data were expressed as mean \pm SE. The significance of difference was determined by one-way analysis of variance. All significance testing was based upon a P-value of $<0.05$. For assessment of the size and intensity of yolk in zebrafish, unpaired t-test was used to measure difference versus DMSO group $(n=10)$. The statistical significance was based upon a P-value of $\leq 0.05$ or $\leq 0.01$.

\section{Results}

\section{Tanshinone I concentration-dependently inhibits lipid accumulation and reduces TG content in differentiating 3T3-L1 cells}

The protocol for 3T3-L1 preadipocyte differentiation is shown in Fig. 1A. Primarily, we investigated the treatment effect of various concentrations $(1,2.5$, and $5 \mu \mathrm{M})$ of tanshinone I (Fig. 1B) on cell growth (survival) during the differentiation of 3T3-L1 preadipocytes into adipocytes for 8 days using cell count analysis. Treatment with tanshinone I at the concentrations tested did not influence survival of 3T3-L1 cells (Fig. 1C). We next investigated the treatment

(A)

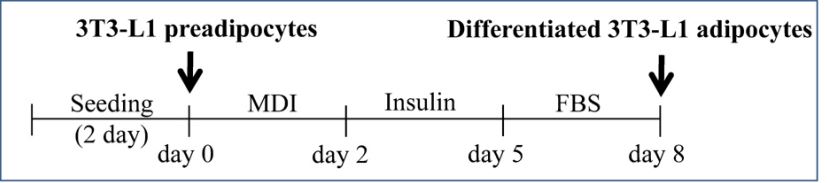

(D)

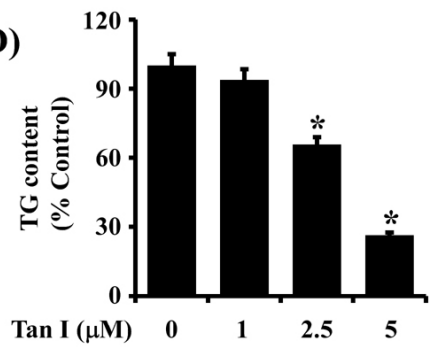

(B)

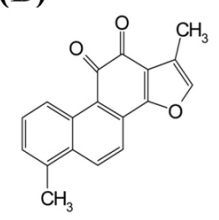

3T3-L1

(C)

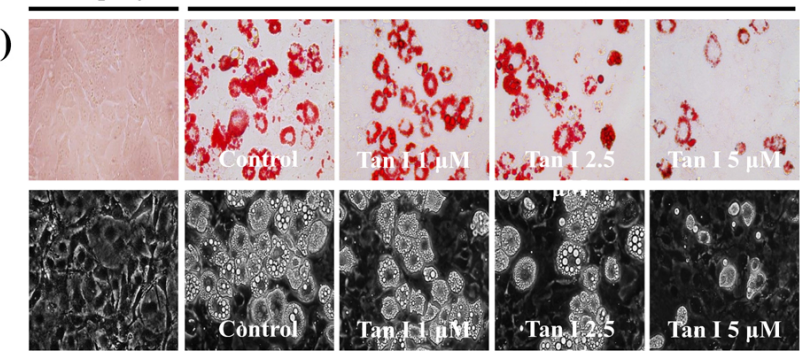

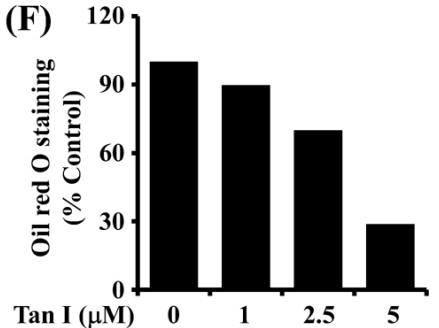

Fig. 1. Effects of tanshinone I on lipid accumulation, TG content, and cell survival during 3T3-L1 preadipocyte differentiation. (A) Protocol for 3T3-L1 preadipocyte differentiation. (B) is the chemical structure of tanshinone I. (C) 3T3-L1 preadipocytes were grown under the above-mentioned 3T3-L1 preadipocyte differentiation condition in Fig. 1A. On day 8, the number of surviving cells in vehicle control or tanshinone I-treated 3T3-L1 preadipocytes was measured by trypan blue dye exclusion. Data are mean $\pm S E$ of three independent experiments, each done in triplicate. ${ }^{*} \mathrm{P}<0.05$ vs. control (no chemical). (D) Quantification of the cellular TG content in vehicle control or tanshinone I-treated 3T3-L1 preadipocytes on day 8 by AdipoRed assay. Data are mean \pm SE of three independent experiments, each done in triplicate. ${ }^{*} \mathrm{P}\langle 0.05$ vs. control (no chemical). (E) Measurement of the cellular lipid (lipid droplets) in 3T3-L1 preadipocytes (undifferentiated) or differentiated adipocytes on day 8 of differentiation in the absence (vehicle control; DMSO, $0.1 \%$ ) or presence of tanshinone I at the indicated concentrations by Oil red $O$ staining ( $\times 400)$ (upper panels). Phase-contrast images were also taken after the treatment (lower panels). (F) Quantification of the Oil red $O$ staining in vehicle control or tanshinone I-treated 3T3-L1 preadipocytes on day 8 by Image J. MDI: $0.5 \mathrm{mM}$ IBMX, $0.5 \mu \mathrm{M}$ dexamethasone, and $5 \mu$ $\mathrm{g} / \mathrm{mL}$ insulin, FBS: fetal bovine serum, Tan I: tanshinone I, TG: triglyceride, SE: standard error, DMSO: dimethyl sulfoxide. 
effect of various concentrations of tanshinone I on intracellular TG content in 3T3-L1 cells on day 8 of differentiation using an AdipoRed assay. As shown in Fig. 1D, tanshinone I treatment concentration-dependently reduced cellular TG content during 3T3-L1 preadipocyte differentiation. We further investigated the treatment of tanshinone I at 1, 2.5, and $5 \mu \mathrm{M}$ on lipid accumulation during the differentiation of 3T3-L1 preadipocytes into adipocytes using Oil red $\mathrm{O}$ staining. Treatment of the preadipocytes with tanshinone I for 8 days markedly reduced the amounts of LDs in a concentration-dependent manner (Fig. 1E, upper panels). Evidently, treatment with $5 \mu \mathrm{M}$ tanshinone I most strongly blocked the accumulation of LDs. In addition, the tanshinone I-mediated inhibition of LD accumulation in differentiating 3T3-L1 cells was observed by light microscopy (Fig. 1E, lower panels). Quantification of the Oil red O staining in vehicle control or tanshinone I-treated 3T3-L1 preadipocytes on day 8 was obtained by Image J (Fig. 1F). Since $5 \mu \mathrm{M}$ of tanshinone I exhibited the most strong inhibitory effect on lipid accumulation and TG content with no cytotoxicity, we chose this concentration for further studies.

\section{Tanshinone I decreases C/EBP- $\alpha$, PPAR- $\gamma$, and phosphorylation levels of STAT-3 in differentiating 3T3-L1 cells}

To gain a better understanding of the mechanisms associated with the anti-adipogenic effect of tanshinone I, we next measured the expression and phosphorylation levels (activity) of adipogenic transcription factors, $\mathrm{C} / \mathrm{EBP}-\alpha$, PPAR- $\gamma$, and STAT-3/5, in differentiating 3T3-L1 cells treated with vehicle control or tanshinone I $(5 \mu \mathrm{M})$ using Western blotting and RT-PCR experiment. Tanshinone I considerably repressed protein (Fig. 2A) and mRNA (Fig. 2B) expressions of $\mathrm{C} / \mathrm{EBP}-\alpha$ and PPAR- $\gamma$, notably on days 5 and 8 . Furthermore,

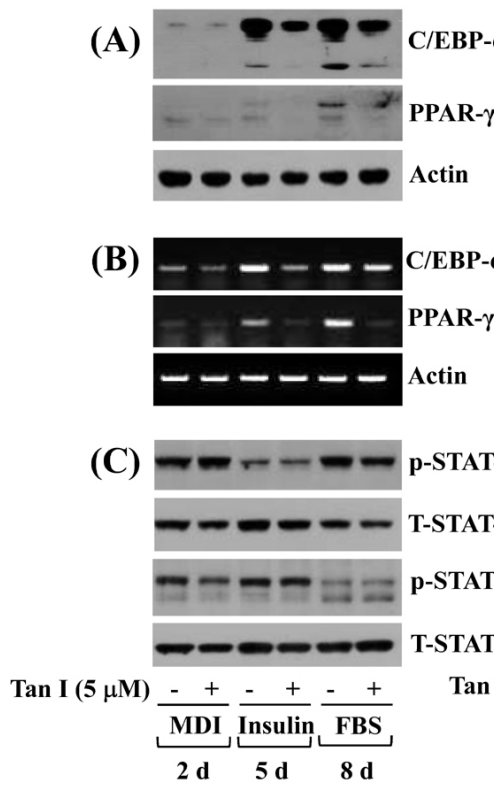

(D)

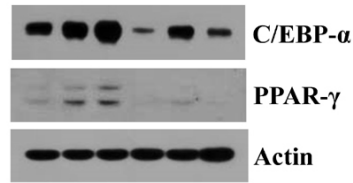

(E)

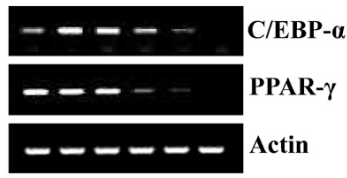

p-STAT-5 (F)

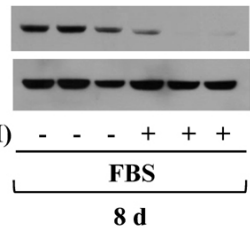

p-STAT-3
(G)

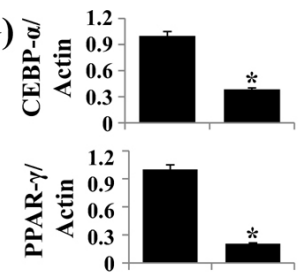

(H)

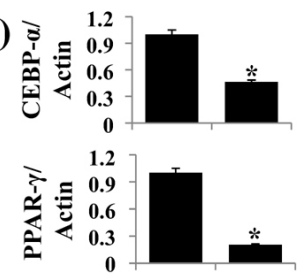

T-STAT-3

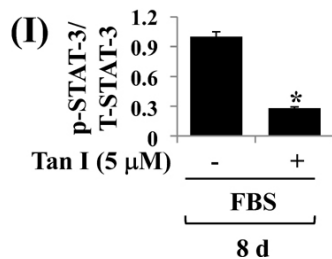

Fig. 2. Effect of tanshinone I on expression and/or phosphorylation of C/EBP- $\alpha$, PPAR- $\gamma$, and STAT-3/5 in differentiating 3T3-L1 cells. (A-C) 3T3-L1 preadipocytes were induced to differentiate with induction medium in the absence (vehicle control, $0.1 \%$ ) or presence of tanshinone I, and harvested at day 2, 5, and 8, respectively. Cellular protein and mRNA at the indicated time point were extracted and analyzed by Western blot (A, C) and RT-PCR (B) analysis, respectively. (D-F) Western blot (D,F) and RT-PCR (E) analysis in triplicate experiments on day 8, respectively. $(G)$ and $(H)$ are the densitometry data of (D) and (E), respectively. (I) is the densitometry result of (F). C/EBP- $\alpha$ : CCAAT/enhancer-binding protein- $\alpha$, PPAR- $\gamma$ : peroxisome proliferator-activated receptor- $\gamma$, STAT: signal transducer and activator of transcription, MDI: $0.5 \mathrm{mM} \mathrm{IBMX,} 0.5 \mu \mathrm{M}$ dexamethasone, and $5 \mu \mathrm{g} / \mathrm{mL}$ insulin, FBS: fetal bovine serum, Tan I: tanshinone I, mRNA: messenger RNA, RT-PCR: reverse transcription polymerase chain reaction. ${ }^{*} \mathrm{P}<0.05$ compared to the value of tanshinone I free control at the indicated day. 
although tanshinone I did not inflect the phosphorylation or total expression levels of STAT-3 protein on days 2 and 5 of adipocyte differentiation, it considerably reduced STAT-3 phosphorylation levels without affecting the protein expression levels on day 8 of adipocyte differentiation (Fig. 2C). Tanshinone I did not reduce phosphorylation and total expression levels of STAT-5 protein on days 2, 5, and 8 of adipocyte differentiation. Triplicate experiments confirmed the capability of tanshinone I to strongly reduce the protein and mRNA expressions of C/EBP- $\alpha$ and PPAR- $\gamma$, and the phosphorylation levels of STAT-3 on day 8 of differentiation (Fig. 2D-F). The densitometry data of Fig. 2D, 2E, and 2F are shown in Fig. 2G, $2 \mathrm{H}$, and $2 \mathrm{I}$, respectively.

\section{Tanshinone I reduces FAS, perilipin A, and leptin in differentiating 3T3-L1 cells}

We next examined the effects of tanshinone I on expressions of FAS (a lipogenic enzyme), perilipin A (an
LD-interacting protein), and leptin (an adipokine) during 3T3-L1 preadipocyte differentiation. Tanshinone I markedly reduced the protein (Fig. 3A) and mRNA (Fig. 3B) levels of FAS and perilipin A especially on days 5 and 8 of adipocyte differentiation. In addition, tanshinone I strongly reduced cellular levels of the leptin mRNA transcripts on days 5 and 8 . Triplicate experiments confirmed the inhibitory potential of tanshinone I on the protein expressions of FAS and perilipin A (Fig. 3C) and the mRNA expressions of FAS, perilipin A, and leptin (Fig. 3D) on day 8 of adipocyte differentiation. The densitometry data of Fig. 3C and $3 \mathrm{D}$ are shown in Fig. $3 \mathrm{E}$ and $3 \mathrm{~F}$, respectively.

\section{Tanshinone I differentially regulates the protein} phosphorylation and total expression of AMPK, ACC, and LKB-1 in differentiating 3T3-L1 cells

AMPK is a major regulator of energy metabolism ${ }^{15,16)}$, and its activation (phosphorylation) leads to inhibition of

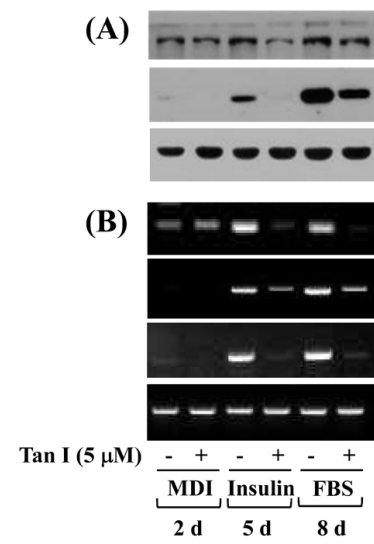

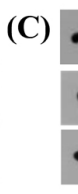

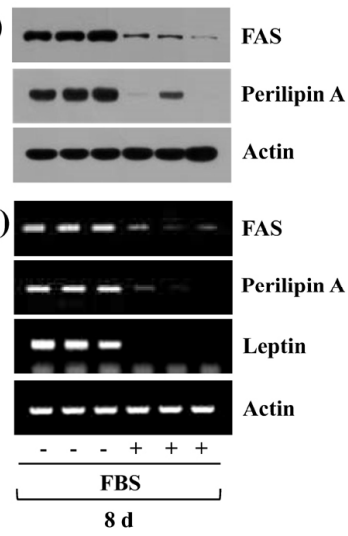

(E)
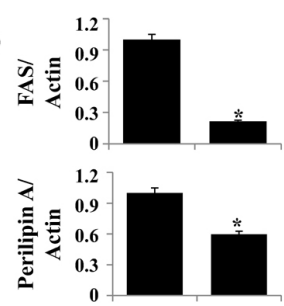

(F)

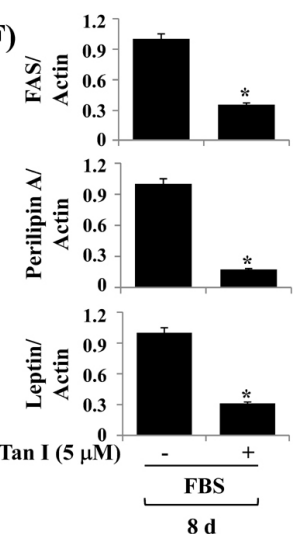

Fig. 3. Effect of tanshinone I on expression of FAS, perilipin A, and leptin in differentiating 3T3-L1 cells. (A, B) 3T3-L1 preadipocytes were induced to differentiate with induction medium in the absence (vehicle control, $0.1 \%$ DMSO) or presence of tanshinone I, and harvested at day 2, 5, and 8, respectively. Cellular protein and mRNA at the indicated time point were extracted and analyzed by Western blot (A) and RT-PCR (B) analysis, respectively. (C, D) Western blot (C) and RT-PCR (D) analysis in triplicate experiments on day 8, respectively. (E) and (F) are the densitometry data of (C) and (D), respectively. FAS: fatty acid synthase, MDI: $0.5 \mathrm{mM}$ IBMX, $0.5 \mu \mathrm{M}$ dexamethasone, and $5 \mu \mathrm{g} / \mathrm{mL}$ insulin, FBS: fetal bovine serum, Tan I: tanshinone I, DMSO: dimethyl sulfoxide, mRNA: messenger RNA, RT-PCR: reverse transcription polymerase chain reaction. ${ }^{*} \mathrm{P}<0.05$ compared to the value of tanshinone I free control at the indicated day. 
adipogenesis ${ }^{28)}$. We thus probed whether tanshinone I affects the expression and/or phosphorylation of AMPK during 3T3-L1 preadipocyte differentiation. In the absence of tanshinone I, there was no or weak AMPK phosphorylation during 3T3-L1 preadipocyte differentiation (Fig. 4A). Tanshinone I slightly increased AMPK phosphorylation on day 2, while it caused a marked increase in AMPK phosphorylation on day 8 of adipocyte differentiation. Tanshinone I did not affect total AMPK protein levels on days 2, 5, or 8 of adipocyte differentiation. ACC is a known downstream effector of AMPK and is involved in the biosynthesis of fatty acids ${ }^{14)}$. We next measured the effect of tanshinone I on the expression and/or phosphorylation levels of ACC during 3T3-L1 preadipocyte differentiation. There was a time-dependent increase in ACC phosphorylation during 3T3-L1 preadipocyte differentiation in the absence of tanshinone I. However, total protein levels of ACC declined on days 2 and 8 of differentiating 3T3-L1 cells treated with tanshinone I, compared with the vehicle-treated cells. Given that LKB-1 is a known upstream activator of AMPK phosphorylation $^{29)}$, we next sought to explore whether LKB-1 protein is expressed and activated (phosphorylated) in differentiating 3T3-L1 cells and is regulated by tanshinone I. Notably, high levels of phosphorylated LKB-1 were observed on day 2 but greatly declined on days 5 and 8 of differentiating 3T3-L1 cells. Furthermore, there was a time-dependent increase in the total protein expression of LKB-1 during 3T3-L1 preadipocyte differentiation. Tanshinone I did not affect the phosphorylation or total protein levels of LKB-1 on days 2, 5, and 8 of adipocyte differentiation. In addition, although there was an enhancement of ACC mRNA on day 2 of differentiating 3T3-L1 cells treated with tanshinone I, there was a reduced expression of ACC mRNA on days 5 and 8 of differentiating 3T3-L1 cells treated with tanshinone I, compared with the mock-treated cells (Fig. 4B). Triplicate experiments further revealed the ability of tanshinone I to largely increase levels of AMPK phosphorylation but down-regulate those of ACC protein and
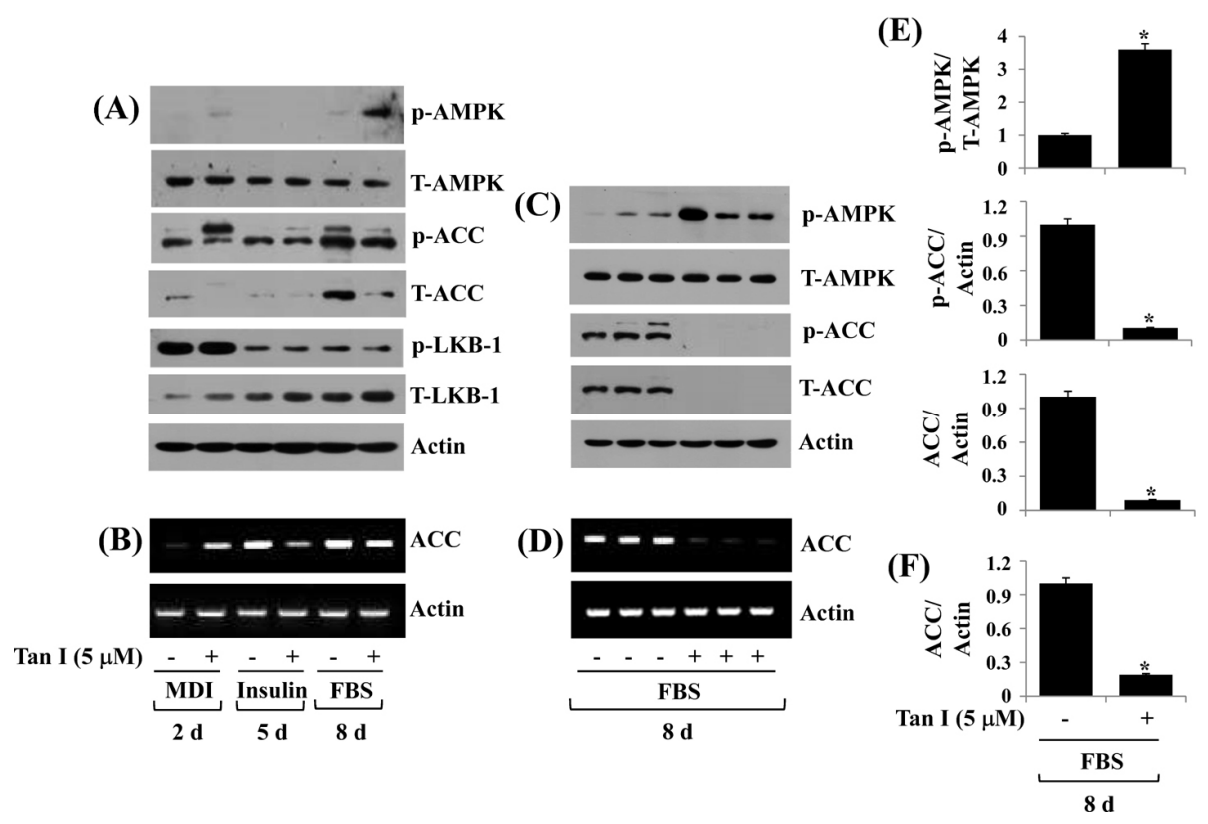

Fig. 4. Effect of tanshinone I on expression and phosphorylation of AMPK, ACC, and LKB-1 in differentiating 3T3-L1 cells. (A, B) 3T3-L1 preadipocytes were induced to differentiate with induction medium in the absence (vehicle control, $0.1 \%$ DMSO) or presence of tanshinone I, and harvested at day 2, 5, and 8, respectively. Cellular protein and mRNA at the indicated time point were extracted and analyzed by Western blot (A) and RT-PCR (B) analysis, respectively. (C, D) Western blot (C) and RT-PCR (D) analysis in triplicate experiments on day 8, respectively. (E) and (F) are the densitometry data of (C) and (D), respectively. AMPK: cAMP-activated protein kinase, ACC: acetyl CoA carboxylase, LKB-1: liver kinase-B1, MDI: 0.5 mM IBMX, $0.5 \mu \mathrm{M}$ dexamethasone, and $5 \mathrm{\mu g} / \mathrm{mL}$ insulin, FBS: fetal bovine serum, Tan I: tanshinone I, DMSO: dimethyl sulfoxide, mRNA: messenger RNA, RT-PCR: reverse transcription polymerase chain reaction. ${ }^{*} \mathrm{P}<0.05$ compared to the value of tanshinone I free control at the indicated day. 
mRNA on day 8 of differentiation (Fig. 4C and 4D). The densitometry data of Fig. 4C and 4D are shown in Fig. 4E and $4 \mathrm{~F}$, respectively.

\section{Tanshinone I reduces intracellular levels of ATP in differentiating 3T3-L1 cells}

AMPK phosphorylation is induced by changes in cellular adenosine monophosphate (AMP)/ATP ratio ${ }^{30)}$. We thus examined the effect of tanshinone I on intracellular ATP contents in differentiating 3T3-L cells. 2-DG, a glucose mimetic that depletes intracellular ATP levels ${ }^{31)}$, was included as a control. Tanshinone I substantially reduced intracellular levels of ATP on days 2, 5, and 8 of adipocyte differentiation, which was comparable to the reductive effect by 2-DG (Fig. 5A-C).

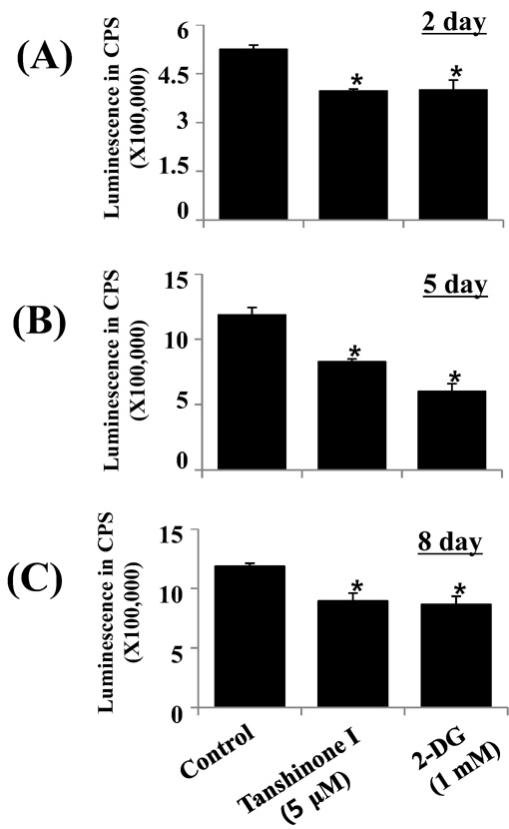

Fig. 5. Effect of tanshinone I on intracellular ATP levels in differentiating 3T3-L1 cells. (A-C) 3T3-L1 preadipocytes were induced to differentiate with induction medium in the absence (vehicle control, $0.1 \%$ DMSO) or presence of tanshinone I or 2-DG, a known ATP depleting agent, and harvested at day 2 $(\mathrm{A}), 5(\mathrm{~B})$, and $8(\mathrm{C})$, respectively. The amounts of intracellular ATP at the indicated time point were measured by a ATP measurement kit. CPS: counts per second, 2-DG: 2-deoxyglucose, ATP: adenosine triphosphate, DMSO: dimethyl sulfoxide. ${ }^{*} \mathrm{P}<0.05$ compared to the value of tanshinone I or 2-DG free control at the indicated day.

\section{Tanshinone I reduces lipid accumulation in the developing zebrafish}

To test its in vivo efficacy, we next investigated the effect of tanshinone I on lipid accumulation in zebrafish using LipidGreen2 staining. To this end, 2 dpf larvae were exposed to vehicle control (DMSO) or tanshinone I at the indicated concentrations $(1,10$, or $100 \mu \mathrm{M})$ for $24 \mathrm{~h}$. The conditioned larvae were washed, anesthetized, and stained with Lipid Green 2 and visualized under bright field and fluorescence. In this study, the reduction of fluorescence was used as an indicator of inhibition of lipid accumulation. Notably, there was a significant decrease in the size of yolk in the group of larvae zebrafish treated with tanshinone I at the doses applied, compared with that in the control group (Fig. 6A). Moreover, there was a significant reduction in size (Fig. 6B and 6C) and intensity (Fig. 6B and 6D) of the fluorescence in the yolk in the group of larvae zebrafish

(A)

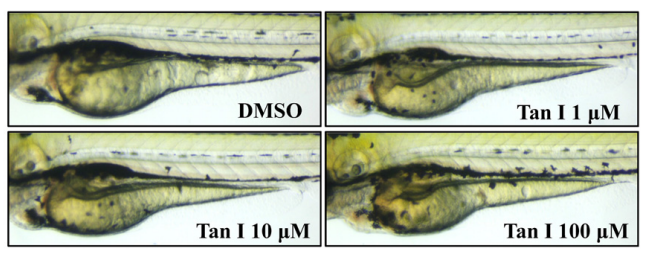

(B)

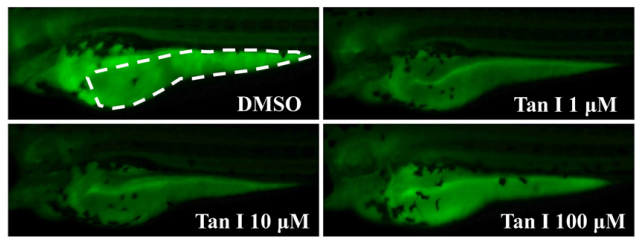

(C)

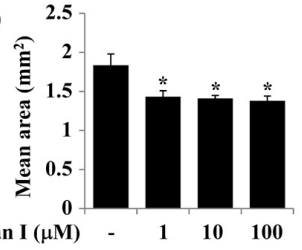

(D) 8

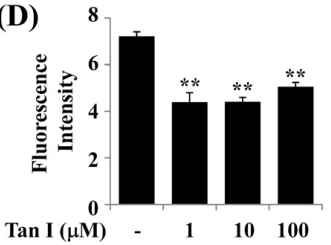

Fig. 6. Effect of tanshinone I on lipid accumulation in zebrafish. Two dpf larvae were exposed in the absence (DMSO) or presence of tanshinone I at the indicated concentrations. After 24 h, 3 dpf larvae were stained with LipidGreen2 and visualized under bright field (A) and fluorescence field (B). (C, D) Size and intensity of yolk (white dash in B) were quantified using the ImageJ software (U.S. National Institutes of Health, Bethesda, MD, USA), respectively. DMSO: dimethyl sulfoxide, Tan I: tanshinone I. Graph bars indicate meantstandard error of the mean. Unpaired t-test was used to analyze difference versus DMSO group, and statistical significance was set 0.05 and $0.01\left({ }^{*} \mathrm{P} \leq 0.05,{ }^{*} \mathrm{P} \leq 0.01\right) . \mathrm{n}=10$. 
treated with tanshinone I at the concentrations tested. Apparently, treatment with tanshinone I even at $1 \mu \mathrm{M}$ was enough to significantly reduce the size and intensity of the fluorescence in the yolk in larvae zebrafish, supporting the potency of tanshinone I to inhibit lipid accumulation in developing zebrafish.

\section{Discussion}

Obesity is associated with the development of type 2 diabetes, insulin resistance, hyperlipidemia, and cancer ${ }^{1)}$. There is also a wealth of information that excessive preadipocyte differentiation (adipogenesis) and the resultant high fat accumulation leads to the development of obesity ${ }^{2,3)}$. Blockers of excessive preadipocyte differentiation and adipogenesis may have therapeutic potential against obesity and related disorders. In this study, we demonstrated firstly that tanshinone I has strong inhibitory effects on lipid accumulation in 3T3-L1 preadipocytes and zebrafish.

It is well documented that adipocyte differentiation is largely controlled by the expression and activity of adipogenic transcription factors C/EBP- $\alpha$, PPAR- $\gamma$, and STAT-3/5 $5^{3-6,32)}$. It has been previously reported that other tanshinones such as tanshinone IIA and cryptotanshinone inhibit adipogenesis in 3T3-L1 cells, and their anti-adipogenic effects are mediated through the reduced expression/activity/phosphorylation levels of C/EBP- $\alpha$, PPAR- $\gamma$, and/or STAT- $3 / 5^{33,34)}$. Of interest, a recent study has demonstrated that tanshinone I has anti-obesity effects on a high fat diet fed mice and anti-adipogenic effects on 3T3-L1 preadipocytes, which are mainly due to inhibition of early stage of differentiation through the attenuation of mitotic clonal expansion via cell cycle arrest, inactivation of $\mathrm{p} 38$ mitogen-activated protein kinase, ERK-1/2, and protein kinase $\mathrm{B}$, and down-regulation of PPAR- $\gamma^{35)}$. However, as of now, little is known about tanshinone I regulation of C/EBP- $\alpha$, PPAR- $\gamma$, and STAT-3/5 during 3T3-L1 preadipocyte differentiation. Here we found that tanshinone I reduced not only the protein and mRNA expressions of C/EBP- $\alpha$ and PPAR- $\gamma$ but also the phosphorylation levels of STAT-3 during 3T3-L1 preadipocyte differentiation. It is thus conceivable that the anti-adipogenic effect of tanshinone I on 3T3-L1 cells is closely attributable to down-regulation of the expression and phosphorylation levels of C/EBP- $\alpha$, PPAR- $\gamma$, and STAT-3. It will be interesting to investigate, in the future, how tanshinone I regulates not only the transcription of $\mathrm{C} / \mathrm{EBP}-\alpha$ and PPAR- $\gamma$ but also the phosphorylation of STAT-3 using pharmacological inhibition study and phosphoproteomics (kinome) approaches.

We have recently demonstrated that the cellular levels of FAS and perilipin A are increased during the differentiation of 3T3-L1 preadipocyte into adipocyte ${ }^{22,36}$. In a recent study, it is also demonstrated that tanshinone I inhibits FAS expression in early stage of 3T3-L1 preadipocyte differentiation $^{35)}$. Until now, however, tanshinone I regulation of FAS and perilipin A expression during 3T3-L1 preadipocyte differentiation process is unknown. Considering that FAS is a known lipogenic enzyme responsible for the synthesis of fatty $\operatorname{acid}^{37)}$, and perilipin A interacts with and stabilizes the newly formed LDs during 3T3-L1 preadipocyte differentiation $^{12,38)}$, the present findings that tanshinone I greatly reduces the protein and mRNA levels of both FAS and perilipin A in differentiating 3T3-L1 cells strongly support the notion that down-regulation of FAS and perilipin A may further contribute to the tanshinone I's anti-lipogenic and lipid-lowering effects. The adipose tissue, as an endocrine organ, synthesizes and secretes an array of adipocytokines, including leptin. The role of leptin in obesity and related disorders has been previously reported ${ }^{34)}$. Tanshinone I inhibited leptin mRNA expression in differentiating 3T3-L1 cells, which further supports its potential utility for the prevention and/or treatment of obesity and related diseases in which excessive leptin production is problematic.

AMPK is a key regulator of energy metabolism and balance $^{15,30)}$. It is a heterotrimeric protein kinase consisting of a catalytic $\alpha$ subunit and regulatory $\beta$ and $\gamma$ subunits $^{30)}$. There is accumulating evidence that AMPK activation leads to inhibition of adipogenesis ${ }^{28,39,40)}$. AMPK activation inhibits ATP-consuming anabolic processes while activating ATP-producing catabolic processes ${ }^{16)}$, in part via the phos- 
phorylation of metabolic enzymes, such as $\mathrm{ACC}^{41)}$. It has been previously reported that tanshinone IIA induces activation of AMPK in cancer cells ${ }^{42)}$ and increases AMPK activity in myoblasts and $\mathrm{db} / \mathrm{db}$ mice ${ }^{43)}$. Here we showed that tanshinone I increases AMPK phosphorylation while decreasing ACC phosphorylation/expression. It may therefore be possible that the anti-adipogenic effect of tanshinone I on 3T3-L1 cells is mediated through AMPK activation and ACC down-regulation, which would confer inhibition of ATP-consuming anabolic processes, such as fatty acid synthesis. It is known that activation of AMPK by phosphorylation on T172 within the $\alpha$ subunit is affected by the expression/activity of LKB-1 and/or changes in the AMP/ATP ratio $^{29,30)}$. Tanshinone I did not alter the phosphorylation or total expression levels of LKB-1, but it substantially reduced intracellular ATP levels in differentiating 3T3-L1 cells. These results suggest that tanshinone I's stimulating effect on AMPK phosphorylation is mediated by reducing intracellular ATP levels (or alteration of the AMP/ATP ratio) rather than the LKB-1 pathway.

It is evident that tanshinone I appears to have strong inhibitory effects on differentiation of 3T3-L1 preadipocytes and lipid accumulation in zebrafish presented in the current study and anti-obesity effects on a high fat diet fed mouse model reported previously. However, it should be noted that 3T3-L1 cells, zebrafish, and mouse are the established in vitro and in vivo experimental model systems, and there is also a big difference of anti-adipogenic or lipid-lowering effects by natural substance(s) between mouse preadipocytes and human ones, and among mouse, zebrafish, and human systems. Future studies are thus warranted to investigate if the treatment or administration of tanshinone I will truly inhibit the differentiation of human preadipocytes and the adiposity of obese people or patients.

\section{Conclusion}

Our study shows that tanshinone I has strong anti-adipogenic effects on 3T3-L1 adipocytes and zebrafish, and its lipid-lowering effect in 3T3-L1 cells is mediated through control of the expression and/or phosphorylation levels of C/EBP- $\alpha$, PPAR- $\gamma$, STAT-3, FAS, ACC, perilipin A, and AMPK. These findings advocate that this natural phytochemical tanshinone I may have therapeutic potential against obesity.

\section{Acknowledgements}

The authors sincerely thank Mr. Anil Yadav Kumar for the arrangement and reading of the manuscript. We also deeply thank Drs. Bae MA and Hwang KS (Korea Research Institute of Chemical Technology) for technical help and advice.

\section{References}

1. Kopelman PG. Obesity as a medical problem. Nature. $2000 ;$ 404(6778) : 635-43.

2. Abdelaal M, le Roux CW, Docherty, NG. Morbidity and mortality associated with obesity. Ann Transl Med. $2017 ; 5(7): 161$.

3. Ali AT, Hochfeld WE, Myburgh R, Pepper MS. Adipocyte and adipogenesis. Eur J Cell Biol. 2013 ; 92(6-7) : 229-36.

4. Ghaben AL, Scherer PE. Adipogenesis and metabolic health. Nat Rev Mol Cell Biol. 2019 ; 20(4) : 242-58.

5. Onal G, Kutlu O, Gozuacik D, Dokmeci Emre S. Lipid droplets in health and disease. Lipids Health Dis. 2017 ; 16(1) : 128 .

6. Cao Z, Umek RM, McKnight SL. Regulated expression of three C/EBP isoforms during adipose conversion of 3T3-L1 cells. Genes Dev. 1991 ; 5(9) : 1538-52.

7. Farmer SR. Transcriptional control of adipocyte formation. Cell Metab. 2006 ; 4(4) : 263-73.

8. Lehrke M, Lazar MA. The many faces of PPARgamma. Cell. $2005 ; 123(6)$ : 993-9.

9. Lakshmanan MR, Nepokroeff CM, Porter JW. Control of the synthesis of fatty-acid synthetase in rat liver by insulin, glucagon, and adenosine $3^{\prime}: 5$ cyclic monophosphate. Proc Natl Acad Sci USA. 1971 ; 69(12) : 3516-9.

10. Montalto MB, Bensadoun A. Lipoprotein lipase syn- 
thesis and secretion: effects of concentration and type of fatty acids in adipocyte cell culture. J Lipid Res. 1993 ; 34(3) : 397-407.

11. Beller M, Bulankina AV, Hsiao HH, Urlaub H, Jäckle H, Kühnlein RP. PERILIPIN-dependent control of lipid droplet structure and fat storage in drosophila. Cell Metab. 2010 ; 12(5) : 521-32.

12. Kern PA, Di Gregorio G, Lu T, Rassouli N, Ranganathan G. Perilipin expression in human adipose tissue is elevated with obesity. J Clin Endocrinol Metab. 2004 ; 89(3) : 1352-8.

13. Wolins NE, Brasaemle DL, Bickel PE. A proposed model of fat packaging by exchangeable lipid droplet proteins. FEBS Lett. 2006 ; 580(23) : 5484-91.

14. Duncan RE, Ahmadian M, Jaworski K, Sarkadi-Nagy E, Sul HS. Regulation of lipolysis in adipocytes. Annu Rev Nutr. 2007 ; 27 : 79-101.

15. Greenberg AS, Shen WJ, Muliro K, Patel S, Souza SC, Roth RA, et al. Stimulation of lipolysis and hormone-sensitive lipase via the extracellular signal-regulated kinase pathway. J Biol Chem. 2001 ; 276(48) : 45456-61.

16. Tu Y. Artemisinin-A gift from traditional Chinese medicine to the world (nobel lecture). Angew Chem Int Ed Engl. 2016 ; 55(35) : 10210-26.

17. Sevene E, Banda CG, Mukaka M, Maculuve S, Macuacua S, Vala A, et al. Efficacy and safety of dihydroartemisinin-piperaquine for treatment of Plasmodium falciparum uncomplicated malaria in adult patients on antiretroviral therapy in Malawi and Mozambique: an open label non-randomized interventional trial. Malar J. $2019 ; 18(1): 277$.

18. Qu C, Ma J, Liu X, Xue Y, Zheng J, Liu L, et al. Dihydroartemisinin exerts anti-tumor activity by inducing mitochondrion and endoplasmic reticulum apoptosis and autophagic cell death in human glioblastoma cells. Front Cell Neurosci. $2017 ; 11: 310$.

19. Liu X, Lu J, Liao Y, Liu S, Chen Y, He R, et al. Dihydroartemisinin attenuates lipopolysaccharide-induced acute kidney injury by inhibiting inflammation and oxidative stress. Biomed Pharmacother. $2019 ; 117$ : 109070.

20. Lu P, Zhang FC, Qian SW, Li X, Cui ZM, Dang YJ, et al. Artemisinin derivatives prevent obesity by inducing browning of WAT and enhancing BAT function. Cell Res. 2016 ; 26(10) : 1169-72.

21. Liu Y, Gao S, Zhu J, Zheng Y, Zhang H, Sun H. Dihydroartemisinin induces apoptosis and inhibits proliferation, migration, and invasion in epithelial ovarian cancer via inhibition of the hedgehog signaling pathway. Cancer Medicine. 2018 ; 7(11) : 5704-15.

22. Jang BC. Artesunate inhibits adipogeneis in 3T3-L1 preadipocytes by reducing the expression and/or phosphorylation levels of C/EBP- $\alpha$, PPAR- $\gamma$, FAS, perilipin A, and STAT-3. Biochem Biophys Res Commun. $2016 ; 474(1): 220-5$.

23. Zalatan F, Krause JA, Blask DE. Inhibition of isoproterenol-induced lipolysis in rat inguinal adipocytes in vitro by physiological melatonin via a receptor-mediated mechanism. Endocrinology. 2001 ; 142(9) : 3783-90.

24. Rosen ED, Sarraf P, Troy AE, Bradwin G, Moore K, Milstone DS, et al. PPAR $\gamma$ is required for the differentiation of adipose tissue in vivo and in vitro. Mol Cell. 1999 ; 4(4) : 611-7.

25. Linhart HG, Ishimura-Oka K, DeMayo F, Kibe $T$, Repka D, Poindexter B, et al. C/EBP $\alpha$ is required for differentiation of white, but not brown, adipose tissue. Proc Natl Acad Sci USA. 2001 ; 98(22) : 12532-7.

26. Stephens JM, Morrison RF, Pilch PF. The expression and regulation of STATs during 3T3-L1 adipocyte differentiation. J Biol Chem. 1996 ; 271(18) : 10441-4.

27. Rosen ED, Hsu CH, Wang X, Sakai S, Freeman MW, Gonzalez FJ, et al. C/EBP $\alpha$ induces adipogenesis through PPAR $\gamma$ : A unified pathway. Genes Dev. 2002 ; 16(1) : 22-6.

28. Kershaw EE, Flier JS. Adipose tissue as an endocrine organ. J Clin Endocrinol Metab. 2004 ; 89(6) : 2548-56.

29. Ahima RS. Adipose tissue as an endocrine organ. Obesity (Silver Spring). $2006 ; 14$ Suppl 5 : 242S-9S.

30. Hwang CS, Loftus TM, Mandrup S, Lane MD. Adipocyte differentiation and leptin expression. Annu Rev Cell Dev Biol. 1997 ; 13 : 231-59.

31. Bastard JP, Maachi M, Lagathu C, Kim MJ, Caron M, Vidal $\mathrm{H}$, et al. Recent advances in the relationship between obesity, inflammation, and insulin resistance. Eur Cytokine Netw. 2006 ; 17(1) : 4-12. 
32. Ceddia RB, Somwar R, Maida A, Fang X, Bikopoulos G, Sweeney G. Globular adiponectin increases GLUT4 translocation and glucose uptake but reduces glycogen synthesis in rat skeletal muscle cells. Diabetologia. $2005 ; 48(1): 132-9$.

33. Shehzad A, Iqbal W, Shehzad O, Lee YS. Adiponectin: regulation of its production and its role in human diseases. Hormones (Athens). 2012 ; 11(1) : 8-20.

34. Yadav A, Kataria MA, Saini V, Yadav A. Role of leptin and adiponectin in insulin resistance. Clin Chim Acta Int J Clin Chem. 2013 ; 417 : 80-4.

35. Jung DY, Kim JH, Jung MH. Anti-obesity effects of tanshinone I from Salvia miltiorrhiza bunge in mice fed a high-fat diet through inhibition of early adipogenesis. Nutrients. $2020 ; 12(5)$ : 1242.

36. Friedman J. Fat in all the wrong places. Nature. 2002 ; 415(6869) : 268-9.

37. Miyoshi H, Souza SC, Zhang HH, Strissel KJ, Christoffolete MA, Kovsan J, et al. Perilipin promotes hormone-sensitive lipase mediated adipocyte lipolysis via phosphorylation-dependent and -independent mechanisms. J Biol Chem. 2006 ; 281(23) : 15837-44.

38. Wolins NE, Brasaemle DL, Bickel PE, A proposed model of fat packaging by exchangeable lipid droplet proteins. FEBS lett. 2006 ; 580 : 5484-91.

39. Vingtdeux V, Chandakkar P, Zhao H, Davies P, Marambaud P. Small-molecule activators of AMP-activated protein kinase (AMPK), RSVA314 and RSVA405, inhibit adipogenesis. Mol Med. 2011 ; 17 : 1022-30.

40. Baek JH, Kim NJ, Song JK, Chun KH. Kahweol inhibits lipid accumulation and induces glucose-uptake through activation of AMP-activated protein kinase (AMPK). BMB Rep. 2017 ; 50 : 566-71.

41. Winder WW, Wilson HA, Hardie DG, Rasmussen BB, Hutber CA, Call GB, et al. Phosphorylation of rat muscle acetyl-CoA carboxylase by AMP-activated protein kinase and protein kinase A. J Appl Phys. 1997 ; 82 : 219-25.

42. Yun SM, Jung JH, Jeong SJ, Sohn EJ, Kim B, Kim SH. Tanshinone IIA induces autophagic cell death via activation of AMPK and ERK and inhibition of mTOR and p70 S6K in KBM-5 leukemia cells. Phytother Res. $2014 ; 28$ : 458-64.

43. Hwang SL, Yang JH, Jeong YT, Kim YD, Li X, Lu Y, et al. Tanshinone IIA improves endoplasmic reticulum stress-induced insulin resistance through AMP-activated protein kinase. Biochem Biophys Res Commun. 2013 ; 430 : 1246-52. 